Proceedings of the

$1^{\text {st }}$ International Conference and Exhibition on Future RFID Technologies

Eszterhazy Karoly University of Applied Sciences and

Bay Zoltán Nonprofit Ltd. for Applied Research

Eger, Hungary, November 5-7, 2014. pp. 43-51

doi: 10.17048/FutureRFID.1.2014.43

\title{
WPAN and WBAN-based micro-networks in the light of the used protocols, services and network security*
}

\author{
Alexander Hambalík \\ Institute of Computer Science and Mathematics \\ FEI Slovak University of Technology, Bratislava \\ alexander.hambalik@stuba.sk
}

\begin{abstract}
The paper originated from the outcomes of the recent research which was carried out within the first phase of the VEGA 1/0173/13 project to prepare and launch the research. Besides other activities, we had to make an overview of the new network technologies, their protocols, which define almost every aspect of our lives. Today, we are served by various devices and systems linked with the network. The absence of these devices can change our feelings, comfort, safety and eventually our health state. The proposal of the systems based on such technologies, requires highly experienced and knowledgeable developers. Except for the reliable communication establishment, the significance of the interoperability of the components of these systems has increased which rarely exists on this level in our view. In the first stage we researched, analysed, and tested the software, hardware and protocol solutions with small range but with great mobility using only our limited opportunities. Our paper presents all these above mentioned aspects in compressed and accurate form. The paper also contains useful information about the circuit support for the new equipment which work in the range of 2.4 to 5 $\mathrm{GHz}$ including their new methods and devices of testing.
\end{abstract}

Keywords: wireless networks, mobility, interoperability, WiFi, BlueTooth, ZigBee, IEEE 11073 (X73), ANT +, ETSI EN 300 328, EN 301893

*This research was supported by VEGA 1/0173/13 project Ochrana osobných údajov v mobilných zariadeniach (Privacyon mobile devices). 


\section{Introduction}

The traditional fast cable LAN networks (Local Area Network), MAN (Metropolitan Area Network), WAN (Wide Area Network), based on IP addressing, serve mainly for connections of users and are deployed to serve their requirements by means of client programs. The networks are bound to specific location because of their construction and size, therefore their use is limited.

Present top technologies with wireless solutions eliminate these limits. WPAN and WBAN (Wireless Personal Area Network and Wireless Body Area Network) networks are made for entirely different purposes, as the versions with the optical and metallic connections. They are customized, personalized, containing several nods and by using batteries they can last many years of operation. This type of communication has a small radius and is of the slower M2M (machine to machine) type, so it transforms to a machine to machine connection, sometimes with a connection point for LAN or WAN. These points have a high mobility and if used in a wearable (built-in) form, there will be new opportunities for a long-term and continuous observation and measurments, for health care, body building, playing sports and education.

In the paper we outline properties of the current systems by means of protocols used in such networks and services provided. We pay a considerable attention to mobility, interoperability and security which characterize the new systems. Except for the ZigBee protocol (IEEE 802.15.4) which has been succesfully applied in many areas, we explore more of these protocols according to the indicated criteria. First of all, we examine IEEE 11073 protocol designed for medical devices, originally developed for the fitness and wellness applications. The last one is a part of the factory-installed accessories of many mobile phones of medium or higher prices. For developers, a network support of components is important. These components are used in the realisation of networks, testing of electrical, mechanical properties and compatibility of components in 2.4 to $5 \mathrm{GHz}$.

\section{Why are these networks in the field of our interest?}

One of the most important components of the long-term sustainable development is the continuous maintaining of population health, protection of local and wider environment while preserving the further progress. The new network technologies and protocols that define our lives today play the key roles in each of the above mentioned areas.

The development of the fundaments of our present knowledge was based on two important factors, parameters:

- Summarized multigeneration experience, gained in various fields,

- Technical and technological development since the beginning of time. 
Despite the significant progress, many of processes, including those related to health, have not been recognized sufficiently. Our senses, current technologies, devices are not always suitable as desired to meet our requirements (adequate sensitivity, accuracy, continuous and highly mobile, wearable, resistant against noise and intelligent operation, etc.). Therefore it is still true that the best way to demonstrate the running process is by means of a "black box". Here, we know only the properties of input (e.g. health state) and we can estimate the final state of the output (the symptoms of disease state) but we can barely know the intermediate running processes and their conditions. Similar situation is when we are asked to make a decision. In fact, we do not know what processes affect our decision in given moment. Often the choice of suitable method (the process of cure) is also dependent on some experience and intuition, rather than on a well-known solution of mapped process in detail. Currently, we are searching for missing technologies because they can lead to new ways of development and daily life (such as transport, medicine, education, mass media, etc.). Measurements and monitoring create the fundaments of our knowledge and having the network connection to obtain the information is the essential condition today.

\section{The measurement of required parameters by means of intelligent sensors}

It is not an easy task because they can be measured only rarely. Most of the parameters are measured indirectly. The recently developed devices use the short-range electromagnetic waves and are powered by batteries with low consumption. Network (WPAN, WBAN) up to several thousands of these units can be used for M2M (machine to machine) wireless connection, with very long battery lifespan (sometimes for several years). Their construction makes it relatively easy to integrate nods into the clothing.

From the point of the portable/wearable systems, the great forward step in the TDK technological novelties was made. High stability SAW and BAW technology uses the integrated circuits. In the power amplifiers, made 200 microns thick layer filters with the base of TFAP (Thin Film Acoustic Package) allows to make the ultra-thin radio frequency $(\mathrm{RF})$ modules, with only $0.8 \mathrm{~mm}$ thickness.

\section{Some important properties of the PAN, WPAN and WBAN networks}

We can summarize those properties as follows:

- The PAN networks are made for one person and luckily the networks have only a few meters range which is bridged by conductors, which we can connect by means of LAN or WAN networks. 
- In case of WPAN and WBAN networks, there is a wireless connection between single nodes and the network elements (sensors, etc.) are mobile. Many nods can operate in a small area.

- SWS (or Smart Wearable Sensors Systems) are developed according to guidelines of consortium. They are developed for 24-hour a day observations of physiological and vital functions, activities and monitoring of individual behavior (mainly for medical purposes).

- The BAN and WBAN networks currently represent the top technology. They are made for one person.

- Sensors are portable and wearable and can stay online for a long time.

- They have star-shaped architecture or special so-called mesh architecture, as selforganizing networks, with several hundreds of intelligent sensors, nods. Often they have the connection point to the WAN and LAN network .

- BAN and WBAN networks can communicate within their own micronetworks or between themselves directly by means of special addressing from the point of the closest layout which pertains to the other networks.

- The nodes in the BAN networks are characterized by maintaining the connection in several meters (free zone, $2,4 \mathrm{GHz}$ ).

- From the network protocols we mostly use ZigBee, IEEE 11073, Bluetooth, $\mathrm{ANT}+$, etc. (WiFi is the least suitable for such tasks) [1], [2], [3].

- The nodes work with the lower transport speed but through several channels without help of a man, using automatically created M2M connection. Nods can maintain one-way or two-way connections.

- They can work with protected and sensitive data with protection (these data reflect the physical and mental state of the observed person very well, its habits, conscious and for some impulses spontaneous reactions, its precise location, reflecting the actual position, etc.).

- The nods (sensors, and all pertaining electronic circuits) are powered by the battery. The circuits have lower consumption, are manageable, and the lithium batteries are long lasting and therefore these WBAN networks can uninteruptedly work for several years.

- WBAN networks can be made up the nods with full or limited functioning (nods are cheaper). 


\section{Possibilities of applications}

The use of BAN networks can be various, but there are places where they are indispensable:

- In general, by means of traditional methods (single or random measurements, life observation of events, or the questionary methods, etc.), it would be almost impossible to realize a short or long-term continuous measurements.

- The contemporary research of parameters of mental and physiological state (motorics, the state of obstruction in the common environment (at home or in the factory), because the measured values can be diametrally different from those measured at home or in the factory.

- At the preparation and rehabilitation processes of sports men and sports women.

- In the mapping of the influence of environment and biological rhythm (seasons of year, sections of day, light, enlightment, noise, purity and pressure of air, humidity, temperature of environment, body temperature, heart rate, etc.).

Such networks are made of the intelligent autonomous sensors, smartphones and systems, in constantly changing environment, which is made of these sensors. The data comming from these systems must be modified (anonymized) to allow scrutiny by the third party. Today, it means a great challenge. By careful proposal and detailed elaboration of results we can know more about the processes which are running at the moment. The proposal of such networks is sophisticated.

Checking of the mechanical and electrical properties is definitely important, but with their massive expansion, their compatibility must be tested as well. The standards will take effect in January 2015 and it will bring a great progress in some areas, also in precision. New extended versions of standards ETSI EN $300328 \mathrm{~V}$ 1.8.1 (2.4 GHz) and EN $301893(5 \mathrm{GHz})$ are already harmonized with R\& TT guidelines of the European Commission. The older versions of ETSI EN 300328 $\mathrm{V}$ 1.7.1 defined mainly those levels (parameters) that should comply, but have not defined the test methods. In addition, a new extended version eliminates the above mentioned shortcomings.

The first new tester under the conditions of ETSI suitable for testing of newly developed devices is R\&S TS 8997 equipment. Among the components there is an analogous generator of signals (R\&S SMB 100A) vector generator of signals (R\&S SMBV 100A), the switching and control units (R\&S OSP 120) with the open-ended system. The additional units of the software (R\&S EMC 32) are modules for measurements of the EMC (R\&S EMC 32-K97 and K975-K971). The measurements for the norm ETSI EN $300328>=1 \mathrm{MHz}$ sampling are rastered by $30,000,000$ dots. This equipmemt can test the device with more antennas (for instance MIMO - Multiple Input Multiple Output System), frequency hopping (FHSS - Frequency Hopping Spread Spectrum or spreaded range (DSSS - Direct Sequence Spectrum) 
using systems. The open system is prepared for the next changes in norms by means of software additions.

\section{What parameters can be measured by sensors}

The sensors can measure the level of glucose, oxygen in blood, the impedance of the skin surface, EKG (ECG/EKG) electrocardiogram units, muscle condition meters (EMG), background radiation meters and other advanced sensors, that are less frequently measured.

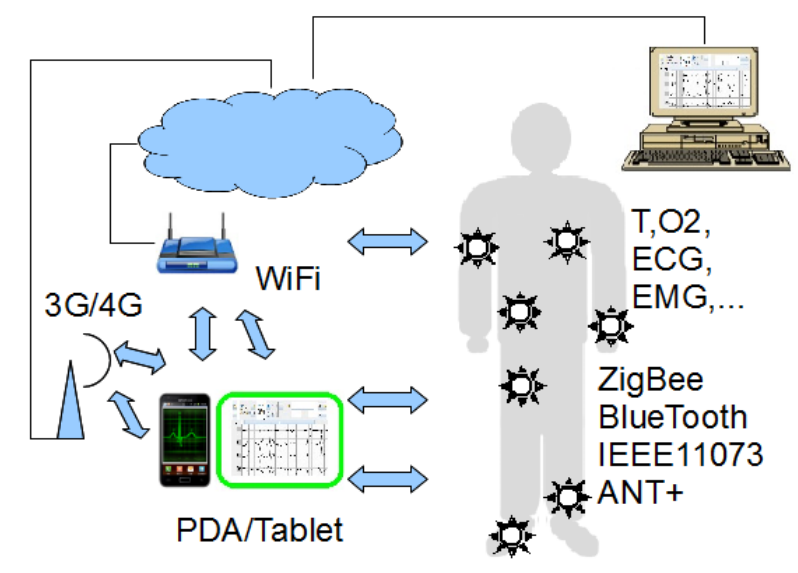

Figure 1: Other development WBAN with multi-way communication network

\section{Used protocols in the light of the security and interoperability}

In addition, except for the IEEE 11073, BlueTooth, the ANT protocol or new $\mathrm{ANT}+$ (mainly fitness) and ZigBee (intelligent buildings) deal with the security of data. From the above mentioned protocols only a few protocols provide the interoperability. Only IEEE 11073 (X73) family of protocols with the protocol IEEE 20601 and IEEE 10XXX, provide the interoperability [5, 6]. The basic set of the family protocols - IEEE 11073 (X73) is designed for the communication with sensors, which were proposed for the given person with computer and mobile phone, supports the interoperability. IEEE 20601 - 2008 describes the communication.

IEEE 10xxx is the family of protocols for each measurement device, such as: 10404 pulse oxymeter [7], 10407 pressure meter monitor, 10408 body temperature thermometers, 10415 body weight meter, 10417 blood glucose meters. 
- Among the singular devices (nods) of the sensor network there is the manageragent relationship.

- The agent node sends a personal (measured) data for the superior managerial nod to which more nods-agents may pertain.

- The nods do not use IP address but use the special locally unique 16-bit identification number (handle).

- The full family of protocols IEEE11073 (X73) enables the connection of the nods in the network and ensures the interoperability between them on the base of object-oriented model.

- The system of network operates according to the three types of models: information model, service model and communication model.

From the new protocols, there is widespread commercially developed ANT+, mainly due to the its factory integration into the smartphones. The ANT + was designed for the smaller systems, for instance for fitness, wellness, sports and leisure applications, but can also occur in medicine. By means of a stable support in the form of integrated circuits (e.g. Texas Instruments non-perspective WL 18xx or a newer one CC25xx, supported by the ultra-low power drain microprocessor MSP430 - in stand-by mode $0.5 \mu \mathrm{A})$, relatively easy to create ANT + connectivity. The basic connection requires $8 \mathrm{~B}$. It means the minimal period for the transmision and low consumption.

Data structure for $\mathrm{ANT}+$ :

[Sync][MsgLength][MsgID][Byte 0]...[Byte N-1][Checksum]

\section{About our results}

Since the start of the research we achieved several results:

- We have explored the technological innovations and new protocols along with their applications in various fields (medicine, fitness, wellness, ANT+, etc.).

- We have made a detailed survey about the important protocols for the internal use (two volumes in range of 500 pages). The first phase of research shortens time of later demanding works.

- In the pilot research we connected the selected types of sensors to the Android/Linux systems.

- We have taken the first steps towards achieving the piconetworks (mobile phones, special sensors).

Unfortunately, our experience discovered that it is not always true that the latest version of the software under development is the most effective. This was confirmed 
by the built-in Android browser, still not fixed that is a serious security flaw. At the time of writing the paper, the latest version of Android 5.0, was problematic. Experiments have shown that in some devices the system was quite unstable. Without any indication the Android was re-booted unexpectedly. Such a self-rebooting can cause serious problems in the field of health care. The accelerated cycles of development with limited range of testing are not able to unveil the serious shortcomings of used solutions. For example, some of the latest models of the leading producer of the smartphones show the constructional flaws that the devices during wearing could be permanently deformed or even broken. These mistakes in some occasions might pose a risk to human life.

\section{Conclusion}

We have tried to outline the possibilities of constructing WPAN and WBAN networks. To outline the technical and other properties, limited fields of use, for instance, the health care, we dealt with other areas as well. Because the development linked with the research takes relatively short time, except for the experiences gained in the field, we presented the experience obtained from the literature, in order to create a real view on networks. We would like to draw your attention to the new opportunities that are linked with these technologies and may open up the areas of research, which will be impossible to research without wearable systems. Most probably it would take some time to gain the sufficient experience from the technical realisation and application. The experience will pave the way for a more complex understanding of the processes which were with our present means unavailable. By means of experience we will create such methods which reduce the workload of parties involved, for instance in the processes of health care and education.

\section{References}

[1] Noueined, J., Diemer, R.Comparing Bluetooth HDP and SPP for Mobile Health Devices, Conference: International Conference on Body Sensor Networks, (2010).

[2] ANT, THIS IS ANT - the Wireless Sensor Network Solution, [11.12.2014]<http: //www.thisisant.com>, (2014).

[3] ZigBee, The Open, Global Wireless Standard for Connecting Everyday Devices, [11.12.2014]<http://zigbee.org/zigbee-for-developers $>$, (2014).

[4] Schmitt, L., Falck, T., Wartena, F., Simons, D.Novel ISO/IEEE 11073 Standards for Personal Telehealth Systems Interoperability, Joint Workshop on High Confidence Medical Devices, (2007).

[5] Schrenker, R. Cooper, T. „Building the Foundation for Medical Device Plug-andPlay Interoperability", Medical Electronics Manufacturing, (2001).

[6] IEEE STd 11073-20601 ${ }^{\mathrm{TM}}-2008$ Health informatics - Personal health device communication - Part 20601: Application profile - Optimized Exchange Protocol, (2008). 
[7] ISO/IEEE 11073 -10404:2010(E)Health informatics - Personal health device communication - Part 10404: Device specialization - Pulse oximeter,(2010). 